\title{
First-Principles Study of Fer romagnetism in Epitaxial Si-Mn Thin Films on Si(001)
}

\author{
Hua Wu, Mahbube Hortamani, Peter Kratzer, and Matthias Scheffler \\ Fritz-Haber-Institut der Max-Planck-Gesellschaft, Faradayweg 4-6, D-14195 Berlin, Germany
}

(Received 27 January 2004; published 10 June 2004)

\begin{abstract}
Density-functional theory calculations are employed to investigate both the epitaxial growth and the magnetic properties of thin $\mathrm{Mn}$ and $\mathrm{MnSi}$ films on $\mathrm{Si}(001)$. For single Mn adatoms, we find a preference for the second-layer interstitial site. While a monolayer Mn film is energetically unfavorable, a cappingSi layer significantly enhances the thermodynamic stability and induces a change from antiferromagnetic to ferromagnetic order. For higher Mn coverage, a sandwiched Si-Mn thin film (with CsCl-like crystal structure) is found to be the most stable epitaxial structure. We attribute the strong ferromagnetic intralayer coupling in these films to $\mathrm{Mn} 3 d-\mathrm{Si} 3 s 3 p$ exchange.
\end{abstract}

PACS numbers: 75.70.-i, 75.50.Pp

A possible route to spintronics devices involves the injection of spin-polarized current from a ferromagnetic (FM) metal into a semiconductor [1,2]. In this context, FM-metal-semiconductor heterojunctions have attracted considerable attention [3-7]. From the viewpoint of applications, it would be highly desirable to grow welldefined FM films on the most common semiconductor, silicon, in particular on the technologically relevant $\mathrm{Si}(001)$ surface. However, common FM metals (Fe, Co) react strongly with $\mathrm{Si}$, and the resultant silicide films are weakly magnetic or even nonmagnetic (NM), and, thus, unsuitable for spintronics devices. We note that $\mathrm{Mn}$ atoms, in suitable chemical environments, possess sizable magnetic moments. Moreover, both fcc-Mn and some $\mathrm{Mn}-\mathrm{Si}$ compounds are closely lattice matched with the $\mathrm{Si}(001)$ surface. We therefore investigated the suitability of thin films of manganese or its intermetallic $\mathrm{Mn}$-Si compounds for growing FM films on $\mathrm{Si}(001)$. Thus far, there are only a few experimental studies on $\mathrm{Mn} / \mathrm{Si}(111)$ [8-10], and even fewer on $\mathrm{Mn} / \mathrm{Si}(001)$ [11]. Theoretical investigations are lacking.

In this Letter, we present first-principles calculations for pseudomorphic Mn and Mn-Si films on $\mathrm{Si}(001)$, focusing on their atomic structure, stability, and magnetic properties. On the basis of our calculations, we propose that metastable FM MnSi films on $\mathrm{Si}(001)$ can be grown that lend themselves to applications in spintronics: First, we find $\mathrm{Mn}$ atoms to prefer a second-layer interstitial site to sites at the surface or in the third layer. This opens the possibility to grow Si-Mn layered structures with a welldefined, atomically sharp interface on $\mathrm{Si}(001)$ required to obtain high spin injection efficiency [6]. Second, we predict a two-layer $\mathrm{Si}-\mathrm{Mn}$ sandwich structure to display the desired FM metallic behavior.

The calculations are performed using densityfunctional theory, employing the all-electron fullpotential augmented plane-wave plus local-orbital method (APW + LO) [12]. The generalized gradient approximation (GGA) [13] is adopted for the exchangecorrelation potential, since it was demonstrated [14] and confirmed by our test calculations that GGA gives a much better description for bulk $\mathrm{Mn}$ than the local-spin-density approximation. Our GGA calculations for the fcc-Mn give the correct layered antiferromagnetic (AFM) ground state with lattice constant $a=3.59 \AA$ and a local spin moment of $1.9 \mu_{B}$, well comparable with the experimental value of about $2.3 \mu_{B}$ [14]. A metastable FM state emerges when $a$ is increased to $3.77 \AA$, which matches rather well with the calculated surface lattice constant of $\mathrm{Si}(001), 3.87 \AA$.

The $\mathrm{Si}(001)$ surface is modeled by a slab consisting of eight or, in some cases, ten $\mathrm{Si}(001)$ layers, on both sides of which $\mathrm{Mn}$ adatoms, and in some cases also capping-Si atoms, are placed (cf. Fig. 1). Note that $\theta=1 \mathrm{ML}$ (monolayer) coverage of $\mathrm{Mn}$ corresponds to two $\mathrm{Mn}$ adatoms per $(1 \times 1)$ cell (on either side of the slab). Supercells with about $10-11 \AA$ vacuum between the slabs and a lateral $(1 \times 1),(2 \times 1)$, or $(2 \times 2)$ periodicity [15] are used. The

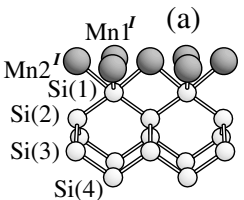

(b)

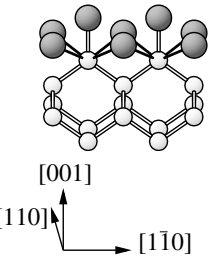

(c)

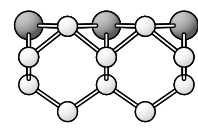

(d)

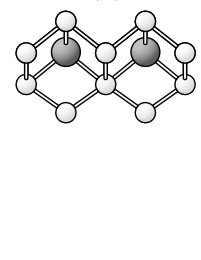

(e) $\mathrm{Si}^{I}$

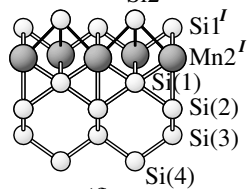

(f) ${ }^{I I}$

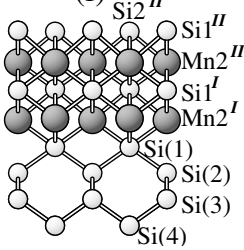

FIG. 1. Perspective or side view of some of the structures considered in the calculations. Black balls show Mn and gray balls $\mathrm{Si}$ atoms. The left column shows 1 ML Mn overlayers on $\mathrm{Si}(001)$, with $\mathrm{Mn}$ in (a) bridge or (b) on-top sites. The middle column shows alloyed overlayer structures containing $0.5 \mathrm{ML}$ $\mathrm{Mn}$, with $\mathrm{Mn}$ in (c) first-layer or (d) second-layer interstitial sites. The right column shows $\mathrm{Si}-\mathrm{Mn}$ sandwich films, containing (e) 1 ML Mn or (f) 2 ML Mn. The thin lines in (b) and (e) represent Mn-Si bonds longer than $2.65 \AA$. 
muffin-tin sphere radii are chosen to be $1.11 \AA$ for both the $\mathrm{Mn}$ and $\mathrm{Si}$ atoms. Converged results are obtained at a cutoff energy for the interstitial plane-wave expansion of 13.8 Ry, due to the APW + LO scheme [12]. A set of $10 \times$ $10 \times 1$ special $\mathbf{k}$ points is used for integrations over the Brillouin zone of the $(1 \times 1)$ cell, and corresponding sets are used for the larger cells [16]. All Mn and Si atoms except for the two central Si layers are relaxed until the calculated atomic force for each of them is smaller than $0.03 \mathrm{eV} / \AA$. Throughout this Letter, formation energies per $(1 \times 1)$ cell are calculated, defined as $E_{\text {form }}=\left(E_{\text {tot }}-\right.$ $\left.\sum_{i} N_{i} \mu_{i}\right) / 2-\gamma_{\mathrm{Si}} A$, where $E_{\mathrm{tot}}, N_{i}$, and $\mu_{i}$ refer to the total energy per $(1 \times 1)$ supercell with surface area $A$, the number of atoms of each chemical type in the cell, and their chemical potentials as calculated from the corresponding bulk materials [17]. $\gamma_{\mathrm{Si}}=84 \mathrm{meV} / \AA^{2}$ is the surface energy of the clean, reconstructed $\mathrm{Si}(001)$ surface. We perform spin-polarized calculations where local magnetic moments are aligned along the surface normal, either in FM or AFM order.

Since experimental data about the atomic structure of thin $\mathrm{Mn} / \mathrm{Si}(001)$ films are not available, we consider plausible epitaxial structures derived from bulk phases of $\mathrm{Mn}$ and $\mathrm{Mn}-\mathrm{Si}$ compounds. For elemental Mn films, the fcc structure is investigated, with the parallel lattice constant equal to that of the $\mathrm{Si}(001)$ substrate. Among the known bulk phases of $\mathrm{Mn}$ silicides, only $\mathrm{Mn}_{3} \mathrm{Si}$ in the $\mathrm{DO}_{3}$ structure appears to be compatible with pseudomorphic thin film growth on $\mathrm{Si}(001)$ (for $\mathrm{Fe}_{3} \mathrm{Si}$, this structure was suggested from a LEED analysis [18]), but is known to be only weakly magnetic [19]. Therefore, as more promising candidates for FM thin films, we propose a (metastable) CsCl-like MnSi epitaxial structure thus far unobserved for bulk Mn silicides [20]. This structure formally results from substitution of part of the Mn atoms either in fcc$\mathrm{Mn}$ or in $\mathrm{Mn}_{3} \mathrm{Si}$ by Si atoms.

Mn adlayer structures. - We first discuss the stability of a dense adlayer of $\mathrm{Mn}$ atoms on $\mathrm{Si}(001)$, defined as $1 \mathrm{ML}-\mathrm{Mn} / \mathrm{Si}(001)$. The symmetry of the $(1 \times 1)$ cell allows for two different structures, either with $\mathrm{Mn}$ in bridge sites [cf. Fig. 1(a)], or in on-top sites [cf. Fig. 1(b)] relative to the bulk-terminated $\mathrm{Si}(001)$ substrate. The latter one is found to be less stable; i.e., its $E_{\text {form }}$ is higher by $0.51 \mathrm{eV}$. Motivated by the lower $E_{\text {form }}$ of the bridging structure, we take it as a starting point for placing a second and third ML Mn adlayer to model fcc-Mn/ $\mathrm{Si}(001)$. It can be seen in Table I that $E_{\text {form }}$ for Mn overlayers increases monotonously with the Mn coverage and is much higher than that of the $\mathrm{Si}(001)$ surface (reference zero energy). We therefore conclude that the growth of $\mathrm{Mn}$ overlayers on the $\mathrm{Si}(001)$ surface is energetically rather unfavorable. Consequently, pure Mn films must be discarded, and we turn to $\mathrm{Mn}-\mathrm{Si}$ compound structures.

$M n$-Si alloy structures.-We start by establishing that the most favorable absorption position of a single $\mathrm{Mn}$ atom on the $p(2 \times 2) \mathrm{Si}(001)$ surface is in a second-layer interstitial site below the Si dimer. Both the most stable
TABLE I. Formation energies $[\mathrm{eV}$ per $(1 \times 1)$ cell] of films grown by depositing $\theta=0.5-3 \mathrm{ML}$ of $\mathrm{Mn}$ on $\mathrm{Si}(001)$. The superscripts refer to Fig. 1. For the items marked with "x," the self-consistent calculation does not support a FM state.

\begin{tabular}{cccccccr}
\hline \hline & \multicolumn{3}{c}{ Overlayer } & \multicolumn{2}{c}{ Capped by Si } & \multicolumn{2}{c}{ Si-Mn sandwich } \\
$\theta$ & FM & AFM & NM & FM & AFM & FM & AFM \\
\hline 0.5 & $0.77^{\mathrm{c}}$ & $0.81^{\mathrm{c}}$ & $1.64^{\mathrm{c}}$ & $0.68^{\mathrm{d}}$ & $0.65^{\mathrm{d}}$ & & \\
1 & $2.05^{\mathrm{a}}$ & $1.86^{\mathrm{a}}$ & $3.86^{\mathrm{a}}$ & $0.62^{\mathrm{e}}$ & $0.76^{\mathrm{e}}$ & $0.62^{\mathrm{e}}$ & $0.76^{\mathrm{e}}$ \\
2 & $\mathrm{x}$ & 2.03 & 2.72 & $\mathrm{x}$ & 0.53 & $-0.40^{\mathrm{f}}$ & $-0.37^{\mathrm{f}}$ \\
3 & $\mathrm{x}$ & 2.29 & 3.80 & $\mathrm{x}$ & 0.98 & -1.47 & -1.53 \\
\hline \hline
\end{tabular}

positions on the surface and the third-layer interstitial site are higher in energy by 1.0 and $0.8 \mathrm{eV}$, respectively. The latter finding shows that subsurface Mn has to overcome a notable energy barrier to diffuse into the Si bulk. In addition, we verify that a deposited $\mathrm{Mn}$ atom can easily reach the subsurface site. As one possible pathway, we consider dynamic breaking of a $\mathrm{Si}$ dimer on the $\mathrm{Si}(001)$ surface by an arriving $\mathrm{Mn}$ atom, followed by lowering of the $\mathrm{Mn}$ atom into the interstitial site right below the $\mathrm{Si}$ dimer. We find that the energy along this pathway decreases monotonously, apart from a small barrier of $0.3 \mathrm{eV}$ when breaking the dimer, compared to the activation energy of Mn diffusion in bulk Si of $0.63 \mathrm{eV}$ [21]. For growth temperatures lower than $900{ }^{\circ} \mathrm{C}$, when dissolution of $\mathrm{Mn}$ in the Si bulk can be neglected [21], our findings suggest the formation of a Mn film capped by a Si layer. A complete layer [cf. Fig. 1(e)] would act as a barrier for further silicide formation, due to the high stability of $\mathrm{Mn}$ at the second-layer interstitial site. However, it should be possible to grow thicker films by codeposition of $\mathrm{Mn}$ and $\mathrm{Si}$.

From the results of our calculations, collected in Table I, we derive the following structural trends.

Structures capped by a Si layer are generally more stable than Mn surface layers, due to a reduction of the number of dangling bonds ( $\mathrm{Mn}$ and $\mathrm{Si}$ atoms form covalent bonds due to their almost identical electronegativity). Moreover, the capping-Si atoms reach fourfold coordination [see, e.g., Fig. 1(e)], and thus are rather stable. Moving them to twofold coordinated bridge positions results in an increase of $E_{\text {form }}$ from 0.62 to $1.42 \mathrm{eV}$.

Layered structures, i.e., occupation of a (001) plane by a single species, are preferred over mixed occupation of layers, e.g., by $0.28 \mathrm{eV}$ for $1 \mathrm{ML} \mathrm{Mn}$, which indicates the possibility to grow silicide layers with atomically sharp interfaces to $\mathrm{Si}(001)$.

Sandwich structures, i.e., alternating layers of $\mathrm{Mn}$ and $\mathrm{Si}$, are preferred over stacking of two or more Mn layers. In particular, the two-layer sandwich, Fig. 1(f), is more favorable by $0.93 \mathrm{eV}$ compared to the stacking sequence $\mathrm{Si}-\mathrm{Mn}-\mathrm{Mn} / \mathrm{Si}(001)$.

Thus, we find the one-layer [Fig. 1(e)], two-layer [Fig. 1(f)], and three-layer sandwiches to be the most stable structures overall. The negative $E_{\text {form }}$ for the latter two indicate that they are also stable relative to 
decomposition into the clean $\mathrm{Si}(001)$ surface and elemental bulk Mn. However, the positive $E_{\text {form }}$ of 2.36 (3.08) eV relative to bulk MnSi for the FM 1 ML (2 ML) sandwich suggests that the films are metastable in the presence of incoherent $\mathrm{MnSi}$ islands [20]. In the sandwich films, the $\mathrm{Mn}$ atoms in the interfacial layer are sixfold [or sevenfold, see Fig. 1(e)] coordinated to Si, while all other $\mathrm{Mn}$ are eightfold coordinated. There is an important structural trend when going from the one-layer to the twolayer and the three-layer sandwich: The averaged $\mathrm{Mn}-\mathrm{Si}$ interlayer distance decreases from $1.58 \AA$ in the $\mathrm{Si}-\mathrm{Mn} / \mathrm{Si}(001)$ to $1.50(1.53) \AA$ for the interfacial (subsurface) $\mathrm{Mn}$ layer in $2(\mathrm{Si}-\mathrm{Mn}) / \mathrm{Si}(001)$, to $1.46,1.40$, and $1.48 \AA$ for the interfacial, middle, and subsurface-Mn layers in $3(\mathrm{Si}-\mathrm{Mn}) / \mathrm{Si}(001)$, indicating tighter $\mathrm{Mn}-\mathrm{Si}$ bonds in the thicker sandwiches. Moreover, we predict that the capping-Si (subsurface-Mn) layer shows a strong buckling of $0.43(0.17) \AA$ in $\mathrm{Si}-\mathrm{Mn} / \mathrm{Si}(001)$.

Magnetic properties.-The magnetic order in $\mathrm{Mn}$ silicide films is governed by a delicate balance between nearest and next-nearest neighbor magnetic interactions, which in turn are sensitive to details of the atomic structure. Therefore it is crucial to perform a careful relaxation of the surface in order to make valid assignments of magnetic order. In the present case, the thin films support extended states only in two dimensions. As a result of our calculations, we find that this leads to layered magnetic structures, with much weaker interlayer magnetic coupling. Moreover, we find that finite-size effects in the direction of the surface normal, and the role of capping$\mathrm{Si}$ atoms, become important. The latter effect is illustrated by the switching of the magnetic coupling from AFM to FM when going from an uncapped 1 ML Mn film, Fig. 1(a), to a capped structure, Fig. 1(e). As seen in Tables I and II, the enhanced stability due to the $\mathrm{Si}$ capping layer goes along with reduced spin moments. We investigated the electronic origin of this switching by analyzing the orbital-projected density of states (DOS) shown in Fig. 2. In contrast to the AFM $1 \mathrm{ML}-\mathrm{Mn} /$ $\mathrm{Si}(001)$ film, the $\mathrm{Mn} 3 d$ bands of the $\mathrm{Si}-\mathrm{Mn} / \mathrm{Si}(001)$ are remarkably broadened due to the strong $\mathrm{Si} 3 s 3 p$-Mn $3 d$ hybridization. The two capping $\mathrm{Si}^{\mathrm{I}}$ and $\mathrm{Si}^{\mathrm{I}}$ and the interfacial $\mathrm{Si}(1)$ acquire an induced spin moment antipar- allel to the neighboring $\mathrm{Mn}$ spin. All these findings are in accordance with the itinerant $s p-d$ exchange model [22], mediated by the three hopping channels $\mathrm{Mn} 1^{\mathrm{I}}-\mathrm{Si} 1^{\mathrm{I}}\left[\mathrm{Si} 2^{\mathrm{I}}\right.$, $\mathrm{Si}(1)]-\mathrm{Mn} 2^{\mathrm{I}}$ [Fig. 1(e)] for itinerant electrons. According to this model, an AFM (or, in some cases, FM) coupling between neighboring $\mathrm{Mn}$ and $\mathrm{Si}$ spins gives rise to a net FM coupling between next-nearest neighbor Mn atoms, due to a lowering of the electrons' kinetic energy by itinerant $s p$ - $d$ exchange [22]. The spin polarization of carriers at the Fermi level, $P=[$ DOS(down) DOS(up) $] /[$ DOS(down) + DOS(up) $]$ is about $30 \%$, as evaluated from the spin-resolved total DOS in Fig. 2. In strong contrast, in the $1 \mathrm{ML}-\mathrm{Mn} / \mathrm{Si}(001)$, there is only one $\mathrm{Mn} 1^{\mathrm{I}}-\mathrm{Si}(1)-\mathrm{Mn} 2^{\mathrm{I}}$ hopping channel. As a result, such an $s p-d$ exchange is less effective than the direct $d-d$ exchange. By the latter mechanism, the electronic system can effectively lower its kinetic energy by direct $d$ - $d$ hopping accompanied by $\mathrm{AFM} M 1^{\mathrm{I}}-\mathrm{Mn} 2^{\mathrm{I}}$ coupling. Thus, the interesting AFM-FM switching caused by the Si capping layer can be qualitatively explained. Quantitatively, we calculate the energy difference between FM and AFM ordering to be $71 \mathrm{meV} / \mathrm{Mn}$ atom in the $\mathrm{Si}-\mathrm{Mn} / \mathrm{Si}(001)$ sandwich, indicating rather strong $s p$ - $d$ exchange mediated FM coupling.

Interestingly, for the 2(Si-Mn)/Si(001) [Fig. 1(f)], we find a FM metallic ground state with $P$ of about $50 \%$ (DOS not shown here). The sixfold coordinated interfacial Mn atoms have an averaged spin moment of $1.90 \mu_{B}$, the same as in the FM Si-Mn/Si(001) ground state described above, while the eightfold coordinated subsurface-Mn atoms have a reduced spin moment of $1.11 \mu_{B}$. The $s p$ - $d$ hybridization induces an averaged spin moment of $0.02 \mu_{B}$ at the capping-layer Si atoms, of $-0.07 \mu_{B}$ at the sandwich-layer Si, and of $-0.04 \mu_{B}$ at the first-layer substrate $\mathrm{Si}$. Again, the $s p$ - $d$ exchange mediated FM intralayer coupling is found to be strong: The energy cost to change the two $\mathrm{Mn}$ magnetic moments in the unit cell within the same layer from parallel to antiparallel is $79 \mathrm{meV} / \mathrm{Mn}$ atom. On the other hand, the FM interlayer coupling is weak because of the surface twodimensional character of the itinerant $s p$ - $d$ hybridized electrons, and the energy cost of inducing an interlayer AFM ordering is only $8 \mathrm{meV} / \mathrm{Mn}$. As seen in Table II, the

TABLE II. Atom-resolved spin moments (within muffin-tin spheres, in $\mu_{B}$ ) of the $n(=1,2,3) \mathrm{ML}-\mathrm{Mn} / \mathrm{Si}(001)$ films (upper three rows) and of the $n(\mathrm{Si}-\mathrm{Mn}) / \mathrm{Si}(001)$ sandwiches (lower three rows) at their respective ground states. The columns enumerate the atoms starting from the substrate-Si central layer (left) to surface layer (right). Roman superscripts indicate the Mn layers, or capping and sandwich Si layers, if applicable. A non-negligible spin moment is induced at the Si atoms adjacent to Mn.

\begin{tabular}{|c|c|c|c|c|c|c|c|c|c|c|c|c|c|c|c|c|}
\hline & $\operatorname{Si}(4)$ & $\operatorname{Si}(3)$ & $\operatorname{Si}(2)$ & $\operatorname{Si}(1)$ & $\mathrm{Mn} 2^{\mathrm{I}}$ & $\mathrm{Mn} 1^{\mathrm{I}}$ & $\mathrm{Si} 2^{\mathrm{I}}$ & $\mathrm{Si}^{\mathrm{I}}$ & $\mathrm{Mn} 2^{\mathrm{II}}$ & $\mathrm{Mn} 1^{\mathrm{II}}$ & $\mathrm{Si} 2^{\mathrm{II}}$ & $\mathrm{Si} 1^{\mathrm{II}}$ & $\mathrm{Mn} 2^{\mathrm{III}}$ & $\mathrm{Mn} 1^{\mathrm{III}}$ & $\mathrm{Si} 2^{\mathrm{III}}$ & $\mathrm{Si}^{\mathrm{III}}$ \\
\hline $\mathrm{Mn}$ & -0.007 & -0.015 & -0.023 & -0.089 & -3.614 & 3.410 & & & & & & & & & & \\
\hline $2 \mathrm{Mn}$ & 0.002 & 0.005 & 0.026 & -0.022 & 1.173 & 1.883 & & & -3.189 & -3.298 & & & & & & \\
\hline $3 \mathrm{Mn}$ & 0.000 & -0.003 & 0.018 & -0.041 & 0.927 & 2.108 & & & -1.190 & -0.997 & & & 3.305 & 3.399 & & \\
\hline Si-Mn & -0.004 & -0.010 & 0.016 & -0.069 & 1.645 & 2.159 & -0.060 & & & & & & & & & \\
\hline $2(\mathrm{Si}-\mathrm{Mn})$ & 0.001 & -0.003 & 0.025 & -0.039 & 1.818 & 1.987 & -0.067 & -0.075 & 1.196 & 1.017 & 0.040 & 0.009 & & & & \\
\hline $3(\mathrm{Si}-\mathrm{Mn})$ & -0.002 & -0.005 & 0.020 & -0.039 & 1.578 & 1.909 & -0.027 & -0.037 & -0.198 & -0.091 & 0.033 & 0.031 & -1.043 & -1.094 & -0.033 & -0.056 \\
\hline
\end{tabular}



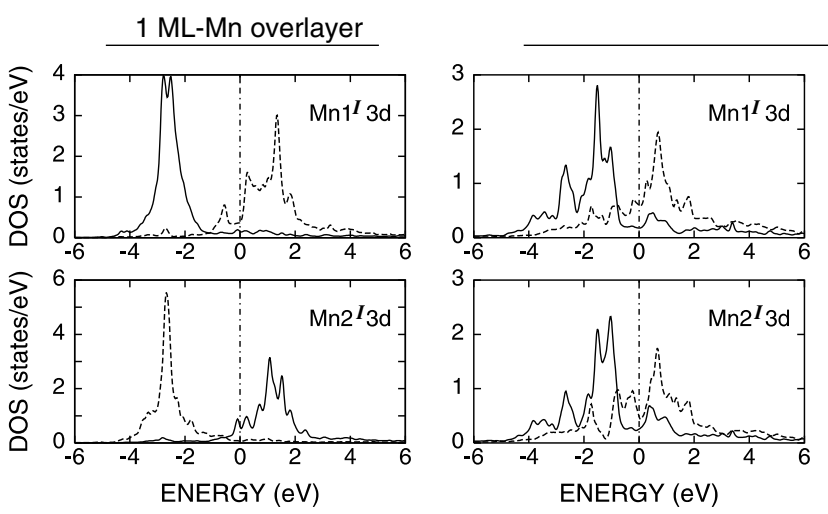

$1 \mathrm{ML}-\mathrm{Mn}$ Si-Mn sandwich
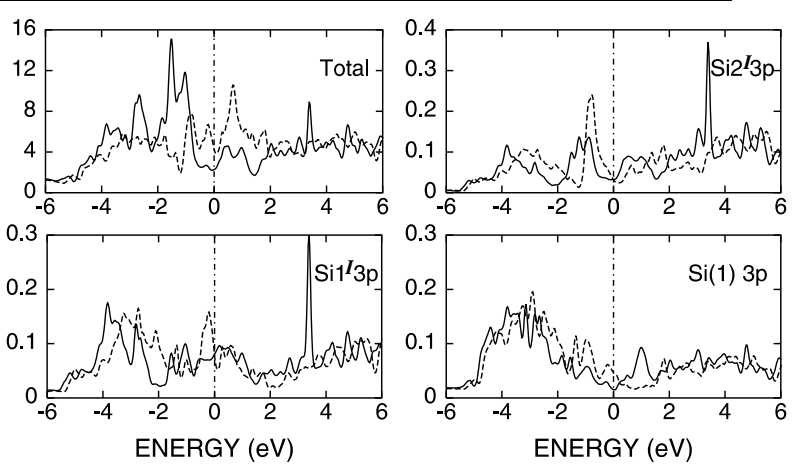

FIG. 2. Projected DOS of the AFM 1 ML-Mn/Si(001) [cf. Fig. 1(a)] and of the FM Si-Mn/Si(001) sandwich [cf. Fig. 1(e)]. The solid (dashed) line refers to the up (down) spin component. The Fermi level is set to zero. In the FM sandwich, the Mn $3 d$ bands are broadened due to strong Mn-Si covalent bonding, and more down-spin states appear at the Fermi level.

Mn spin moments further decrease (and even almost vanish in the middle layer) for the $3(\mathrm{Si}-\mathrm{Mn}) / \mathrm{Si}(001)$. Now, the interlayer coupling between the interfacial and subsurface-Mn layers becomes AFM, partly due to the small but slightly negative spin polarization of the middle Mn layer, which also has the smallest interlayer spacing (see above), and presumably the strongest $\mathrm{Mn}-\mathrm{Si}$ covalency. In a sense, such an AFM (or ferrimagnetic) coupling could, via the intermediate "NM" Si-Mn-Si trilayer complex, have a superexchange origin. Because of the considerable uncompensated spin moment in the $3(\mathrm{Si}-\mathrm{Mn}) / \mathrm{Si}(001)$ film, $P$ is as high as about $30 \%$.

In summary, we predict by means of first-principles calculations that ultrathin $\mathrm{Si}-\mathrm{Mn}$ sandwich films grown pseudomorphically on $\mathrm{Si}(001)$ are ferromagnetic. This calls for experimental studies of their epitaxial growth and applicability for spintronics.

We are grateful to P. Fumagalli, J. Paggel, and H. Lippitz for stimulating discussions. This work was supported by the Deutsche Forschungsgemeinschaft, SFB 290.

[1] G. A. Prinz, Science 282, 1660 (1998).

[2] S. A. Wolf et al., Science 294, 1488 (2001).

[3] G. A. Prinz and J. J. Krebs, Appl. Phys. Lett. 39, 397 (1981).

[4] V. P. LaBella et al., Science 292, 1518 (2001).

[5] H. J. Zhu et al., Phys. Rev. Lett. 87, 016601 (2001).

[6] M. Zwierzycki et al., Phys. Rev. B 67, 092401 (2003).

[7] S. C. Erwin, S.-H. Lee, and M. Scheffler, Phys. Rev. B 65, 205422 (2002).

[8] G. Ctistis et al., J. Magn. Magn. Mater. 240, 420 (2002); U. Deffke et al. (to be published).

[9] S. M. Shivaprasad et al., Surf. Sci. 382, 258 (1997).

[10] S. Kawamoto et al., Surf. Sci. 242, 331 (1991).

[11] J. Wang et al., Appl. Surf. Sci. 113/114, 53 (1997).

[12] P. Blaha et al., Comput. Phys. Commun. 147, 71 (2002).

[13] J. P. Perdew, K. Burke, and M. Ernzerhof, Phys. Rev. Lett. 77, 3865 (1996).
[14] M. Eder, J. Hafner, and E. G. Moroni, Phys. Rev. B 61, 11492 (2000).

[15] A $(2 \times 2)$ supercell is used to calculate the absorption of a single Mn atom (see text). Our calculations show that the dimerization of the $\mathrm{Si}(001)$ surface is lifted for $\theta \geq$ $1 / 2$ ML. Therefore, it is sufficient to consider a $(1 \times 1)$ cell for the $\mathrm{Mn} / \mathrm{Si}(001)$ films in this study.

[16] Careful checks of the numerical accuracy of the present calculations, using higher cutoff energy or more $\mathbf{k}$ points, confirm the convergence of absolute values of $E_{\text {form }}$ within $0.1 \mathrm{eV}$ and a significantly smaller error of only several $\mathrm{meV}$ for the relative stability of structures with the same composition but different geometries and/or magnetic structures.

[17] We calculated $\mu_{\mathrm{Mn}}$ for the AFM fcc-Mn ground state, and corrected by $-0.07 \mathrm{eV} / \mathrm{Mn}$ the energy difference between the ground state $\alpha$-Mn and fcc-Mn [D. Hobbs and J. Hafner, J. Phys. Condens. Matter 13, L681 (2001)].

[18] U. Starke et al., Europhys. Lett. 56, 822 (2001).

[19] C. Pfleiderer et al., Phys. Rev. B 65, 172404 (2002).

[20] Natural bulk MnSi takes on the $P 2{ }_{1} 3$ structure and is a helical magnetic itinerant-electron system [C. Pfleiderer, S. R. Julian, and G. G. Lonzarich, Nature (London) 414, 427 (2001)]. Our calculations for bulk MnSi find the $\mathrm{CsCl}$ structure to be the most stable among the $\mathrm{CsCl}$, NiAs, and $\mathrm{NaCl}$ structures, with heat of formation smaller than that of the $P 2{ }_{1} 3$ structure by $0.25,0.33$, and $1.33 \mathrm{eV}$ per formula unit, respectively. In contrast to the $P 2{ }_{1} 3$, the $\mathrm{CsCl}$ structure with equilibrium lattice constant of $2.79 \AA$ A matches pseudomorphically with $\mathrm{Si}(001)$. Various tetragonal phases of composition $\mathrm{MnSi}_{1.7}$ have a too large unit cell to be relevant for ultrathin films. Moreover, our calculations find the tetragonally strained CsCl-like $\mathrm{MnSi}$ to be a FM metal with spin moment larger than $0.5 \mu_{B} / \mathrm{Mn}$ when the tetragonal distortion exceeds $8 \%$ [i.e., the $c$-axis Mn-Si interlayer distance exceeds $1.48 \AA$, as found in the $\mathrm{Si}-\mathrm{Mn} / \mathrm{Si}(001)$ and $2(\mathrm{Si}-\mathrm{Mn}) / \mathrm{Si}(001)$ films].

[21] D. Gilles, W. Bergholz, and W. Schröter, J. Appl. Phys. 59, 3590 (1986).

[22] J. Kanamori and K. Terakura, J. Phys. Soc. Jpn. 70, 1433 (2001). 